\title{
CONTRIBUIÇÕES DA ABORDAGEM CANADENSE "PRÁTICA DE TERAPIA OCUPACIONAL CENTRADA NO CLIENTE" E DOS AUTORES DA DESINSTITUCIONALIZAÇÃO ITALIANA PARAA TERAPIA OCUPACIONAL EM SAÚDE MENTAL
}

\author{
CONTRIBUTIONS OF THE CANADIAN'S APPROACH \\ "CLIENT-CENTERED OCCUPATIONAL THERAPY" \\ AND OF THE ITALIAN'S DEINSTITUTIONALIZATION \\ AUTHORS FOR THE OCCUPATIONAL THERAPY IN \\ MENTAL HEALTH
}

Elisabete Ferreira Mângia*

\begin{abstract}
MÂNGIA EF. Contribuições da abordagem canadense "prática de Terapia Ocupacional centrada no cliente" e dos autores da desinstitucionalização italiana para a terapia ocupacional em saúde mental. Rev. Ter. Ocup. Univ. São Paulo, v.13, n.3, p.127-34, set./dez. 2002.
\end{abstract}

\begin{abstract}
RESUMO: A construção de novos modelos para a terapia ocupacional em saúde mental se processa em diálogo com o contexto das proposições da Política de Saúde Mental e especialmente com a implementação dos serviços substitutivos. Neste artigo, partindo das proposições da "Terapia Ocupacional Centrada no Cliente" e dos autores da Desinstitucionalização Italiana, buscamos construir um diálogo que possa orientar a adoção de referenciais e diretrizes para as ações técnicas da área. Como pontos centrais de uma nova orientação destacamos: 1. A centralidade da pessoa e do processo de construção/reconstrução de histórias de vida; 2 . O novo tipo de relação cliente-terapeuta-serviço; 3 . O deslocamento do locus da ação da instituição para o território e para as situações da vida real; 4. A nova concepção de projeto terapêutico e a ruptura com a tradicional separação entre prevenção, tratamento e reabilitação; 5. A importância da definição de metas significativas, construídas, explicitadas e compartilhadas com os usuários; 6. O estabelecimento de critérios avaliativos e o desenvolvimento da avaliação continuada dos processos.
\end{abstract}

DESCRITORES: Terapia ocupacional/tendências. Terapia não dirigida. Terapia ocupacional/ recursos humanos.

$\longrightarrow$ processo de formalização dos discursos e da busca de legitimidade da Terapia Ocupacional, enquanto área de produção de saber, tem ocorrido no contexto do diálogo com a diversidade de experiências e proposições presentes nas transformações das Políticas de Saúde, nas formas de ver e dizer sobre a experiência do adoecimento e da deficiência, na projeção dos usuários dos serviços como sujeitos de direito, nas transformações do ensino e da pesquisa, dentre outros aspectos (MÂNGIA, 1999).

Neste trabalho caracterizamos as proposições da

\footnotetext{
Prof $^{\mathrm{a}}$ Dr $^{\mathrm{a}}$ Departamento de Fisioterapia, Fonoaudiologia e Terapia Ocupacional da FMUSP.

Endereço para correspondência: Rua Cipotânea, 51, Cidade Universitária, São Paulo. CEP 05360-160. e-mail: centroto@edu.usp.br
} 
"Terapia Ocupacional Centrada no Cliente" e dos autores da Desinstitucionalização Italiana, que tem nos orientado no contexto da produção de ações de uma nova Terapia Ocupacional no campo da reforma psiquiátrica.

Nossa intenção é a de, no diálogo com essas experiências e proposições, fazer emergir um conjunto de diretrizes que consideramos válidas para orientar nossa prática atual.

\section{A Terapia ocupacional centrada no cliente}

No Canadá, a consolidação teórico-técnica da profissão esteve relacionada a um grande esforço coletivo, coordenado pela Associação Canadense de Terapeutas Ocupacionais, como resposta às pressões sociais e governamentais ligadas à necessidade de estabelecimento de parâmetros que pudessem aferir o impacto e a qualidade dos serviços oferecidos por terapeutas ocupacionais e justificar sua inserção no contexto da reestruturação dos sistemas locais de saúde, especialmente, buscando definir as ações financiáveis pelos recursos públicos.

A discussão sobre o controle dos gastos e aumentos dos custos dos serviços de saúde, a pressão dos consumidores por melhorias dos serviços e controle dos cuidados recebidos, as mudanças nos perfis epidemiológicos, e especialmente o crescente deslocamento dos serviços em direção à comunidade, foram fatores impulsionadores de mudanças na terapia ocupacional que passou a buscar ofertar práticas que realmente satisfizessem às necessidades das pessoas (LAW, 1998).

Em 1979, teve início um processo de trabalho envolvendo grupos de profissionais, reconhecidos no cenário da terapia ocupacional canadense, que elaboraram diretrizes para nortear o conjunto das práticas desenvolvidas pela terapia ocupacional no país e propiciar um campo comum de diálogo e produção teórico-prática, uma vez que a área ainda caracterizase por uma grande dispersão técnica e conceitual e ancorava muitas práticas, quase que exclusivamente, na cultura oral. Como resultado desse esforço, emergiu a orientação da "Terapia Ocupacional Centrada no Cliente" que, inspirada nas formulações de Carl Rogers, privilegia a qualidade da interação entre terapeuta e cliente no processo terapêutico e reconhece o cliente como sujeito na construção dos projetos terapêuticos (LAW et al. 1995).

Law et al. (1995), admitem que essa orientação implica no aprofundamento da reflexão sobre questões que consideramos também fundamentais para o desenvolvimento da Terapia Ocupacional em nosso contexto, são elas: a parceria entre terapeuta e cliente e a distribuição de poder envolvida nessa relação; o deslocamento de um tipo de relação terapeuta-cliente muito diretiva e marcada pelo modelo médico, para uma relação onde terapeuta se coloca como um elemento facilitador; o deslocamento de um modelo de intervenção baseado na diminuição de déficits funcionais, para um modelo mais preocupado com a habilitação segundo metas significativas para o cliente; a importância do ambiente nos resultados da intervenção e o questionamento sobre a utilização de protocolos únicos para avaliação e intervenção que, perdem de vista a singularidade das necessidades de cada pessoa (LAW et al., 1995; MÂNGIA, 1999).

\section{O que é a prática centrada no cliente?}

O processo de formalização dessas diretrizes foi consolidado a partir do trabalho de 3 diferentes grupos que assumiram a tarefa de formalizar guias sobre a concepção, processo e prática da Terapia Ocupacional Centrada no Cliente. Como resultado desse trabalho foram apresentados os 3 volumes dos "Guias da Prática Centrada no Cliente". A primeira comissão iniciou seu trabalho em 1979, que resultou no primeiro volume, que define a estrutura conceitual da prática centrada no cliente. Reconhece, a partir de uma dimensão holística, que a saúde está ancorada no desempenho ocupacional, dotado de sentido para a pessoa e adequado ao seu momento de vida. Define quatro componentes do desempenho: mental, físico, sócio-cultural e espiritual. Genericamente, cada pessoa integra esses componentes para atingir 3 áreas de desempenho ocupacional: autocuidado, produtividade e lazer. Articula, também, a compreensão de que o desempenho de cada um está intrinsecamente articulado às condições ambientais, sociais, econômicas, físicas e culturais da pessoa. $\mathrm{O}$ desempenho resulta dessa interatividade entre indivíduo e contexto.

O segundo volume, resultado do trabalho da segunda comissão, reunida em 1984, foi publicado em 1986. Nele foram definidas as orientações para o processo de terapia ocupacional centrada no cliente. A terapia ocupacional teria como objetivo central "prevenir a incapacidade e promover, manter ou restaurar o desempenho ocupacional", em pessoas cujo desempenho ocupacional está em risco ou já foi rompido" (TOWNSED et al., 1990).

A partir dos guias, a Terapia Ocupacional Centrada no Cliente pode ser definida como:

"uma abordagem que adota a filosofia de respeito e parceria com as pessoas que recebem os cuidados, 
reconhece a autonomia do cliente e a necessidade dele realizar escolhas e tomar decisões de acordo com suas necessidades" (LAW et al., 1995).

\section{As diretrizes do projeto/processo terapêutico da terapia ocupacional centrada no cliente}

A orientação comum define que as práticas sejam desenvolvidas em 7 estágios distintos: 1) encontro entre terapeuta e cliente, processo conjunto de definição de necessidades e objetivos; 2) escolha da abordagem e instrumentos; 3) planejamento consensual; 4) identificação de aspectos que podem contribuir e fazer parte do processo; 5) estabelecimento de um contrato entre as partes envolvidas; 6) colocação o plano em ação; 7) avaliação do processo.

No processo, a noção de cliente deve ser vista de forma ampliada e abarca a própria pessoa atendida, os familiares, sua rede social, ou até cuidadores, quando se trata de situações institucionais.

\section{O primeiro estágio}

O objetivo do primeiro estágio é identificar questões sobre a performance ocupacional do cliente. É a parte avaliativa do processo. Propõem-se que, no diálogo entre terapeuta ocupacional e cliente, este possa falar sobre seu desempenho cotidiano (auto-cuidado, trabalho e lazer) e que o conteúdo de sua narrativa possa ser discutido na perspectiva de buscar definir as questões significativas do desempenho de acordo com as necessidades identificadas pelo cliente ao buscar a ajuda de um serviço ou de profissionais. Nesse processo terapeuta e cliente, juntos, estabelecem, validam e priorizam as áreas de investimento e os objetivos a serem perseguidos ao longo do processo terapêutico. A identificação de necessidades, a partir da narrativa, implica no reconhecimento contextualizado dos objetivos do processo terapêutico o que não ocorre nas formas pré-codificadas de avaliação por meio de questionários ou instrumentos padronizados que enquadram a pessoa naquilo que já vem pré definido como conjunto de necessidades.

\section{Segundo estágio}

Neste estágio, o terapeuta ocupacional escolhe as abordagens e/ou modelos de intervenção, selecionados a partir do quadro avaliativo construído no primeiro estágio. São vários os modelo utilizados na prática canadense: Modelo da Ocupação Humana; Modelo da Pessoa-Ambiente-Performance Ocupacional;
Adaptação Ocupacional; Modelo Canadense da Performance Ocupacional, etc. As múltiplas necessidades da pessoa podem implicar na adoção combinada de diferentes modelos, o que torna o processo bem flexível e plástico, pois se espera que a abordagem teórica ampare o processo e não o defina a priori como se vê nos modelos tradicionais de reabilitação e/ou cuidado. Nessa mesma perspectiva, também se podem escolher modelos apropriados de avaliação.

\section{Terceiro estágio}

Neste estágio, terapeuta ocupacional e cliente devem identificar, a partir das abordagens escolhidas, os aspectos da performance ocupacional e as condições ambientais que podem contribuir na resolução das necessidades definidas no primeiro estágio. Se espera que haja um processo de singularização na maneira de abordar os problemas, dessa forma, uma mesma necessidade pode ter diferentes encaminhamentos para diferentes clientes. Recomenda-se ao terapeuta que evite suposições ou julgamentos apriorísticos e que construa cada passo do processo juntamente com o cliente.

\section{Quarto estágio}

Neste estágio, o cliente e o terapeuta ocupacional identificam as "forças" (habilidades do cliente) e os "recursos" (o cuidador, os familiares, a comunidade, o terapeuta, os recursos do ambiente, etc) que podem ser acionados no processo. Ao terapeuta cabe agir como facilitador e articulador da relação entre as capacidades da pessoa e os recursos acionáveis presentes no ambiente e nas redes sociais. O processo em terapia ocupacional deve possibilitar que os clientes aumentem suas habilidades para a resolução de problemas, de acordo com a singularidade de cada pessoa. Nesse contexto, a parceria entre terapeuta e cliente é um recurso fundamental ao processo, por outro lado o terapeuta deve sempre considerar que o cliente é sempre quem melhor conhece o seu próprio problema. Quando se percebe que o terapeuta e o cliente não conseguem trabalhar juntos de maneira colaborativa, ou o cliente não gosta de trabalhar com o terapeuta, se sugere que outro terapeuta seja escolhido pelo cliente.

\section{Quinto estágio}

Neste estágio, se estabelece um contrato entre o cliente e o terapeuta onde são identificadas as responsabilidades de todas as partes envolvidas. São 
também identificadas as ações que serão realizadas, para isso o terapeuta e o cliente negociam os "resultados alvo" e traçam os planos de ação.

Para estabelecer os resultados alvo e desenvolver os planos de ação é necessário relacionar as questões da performance ocupacional (primeiro estágio), as habilidades do cliente e os recursos do ambiente (quarto estágio) com os desejos e vontades do cliente. O terapeuta ocupacional fornece ao cliente todas as informações necessárias para que ele possa tomar decisões, clareando as ações a serem tomadas por todos envolvidos no processo. Isto promove a confiança do cliente no terapeuta e no processo, fazendo com que o cliente torne-se cada vez mais ativo e assuma mais responsabilidade pela sua saúde.

\section{Sexto estágio}

Nesse estágio, os planos são colocados em ação. Por terem os resultados alvo bem definidos, o cliente e o terapeuta podem ser flexíveis com os métodos que utilizam para alcançá-los. O terapeuta ocupacional acompanha o cliente, orientando, encorajando, auxiliando a transpor barreiras e a construir pontes, incentivando-o a ser cada vez mais ativo no processo de resolução de problemas.

Espera-se que os clientes possam desenvolver um papel cada vez mais ativo na resolução de suas necessidades e que ajam com autonomia, podendo até substituir atividades, repensar os planos e redimensionar os objetivos iniciais, a partir do desenrolar do processo. É importante considerar, também, o significado que as ações adquirem para o cliente. Por exemplo: preparar alimentos pode ser uma atividade indicada para se alcançar objetivos físicos e ter, ao mesmo tempo, significado para o cliente em suas expectativas profissionais, já, empilhar cones pode resultar na melhora da coordenação motora, mas não ter nenhum outro sentido para a pessoa.

\section{Sétimo estágio}

Neste estágio, o processo deve ser avaliado. $\mathrm{O}$ cliente e o terapeuta avaliam os resultados obtidos em relação aos objetivos e necessidades estabelecidas inicialmente. Os resultados podem ter sido total ou parcialmente atingidos, ou mesmo não terem sido atingidos. O importante é que se possa rever o processo conjuntamente e identificar novas questões ou redirecionar as ações para novos alvos e necessidades.

Todo o processo deve ser documentado, o que torna possível avaliar, comparar e decidir pela continuidade ou não do processo.

Finalmente, na Figura 1 são apresentados os aspectos a serem avaliados e considerados na construção dos projetos terapêuticos, de acordo com o preconizado pela abordagem centrada no cliente. $\mathrm{O}$ detalhamento de cada aspecto mereceria discussão específica que não será realizada neste momento. É importante notar que a pessoa é situada em seu contexto micro e macro social e que, independentemente do problema específico a ser trabalhado, a orientação recomenda que o projeto deva sempre contemplar a interação com os demais aspectos pessoais, micro e macro contextuais.

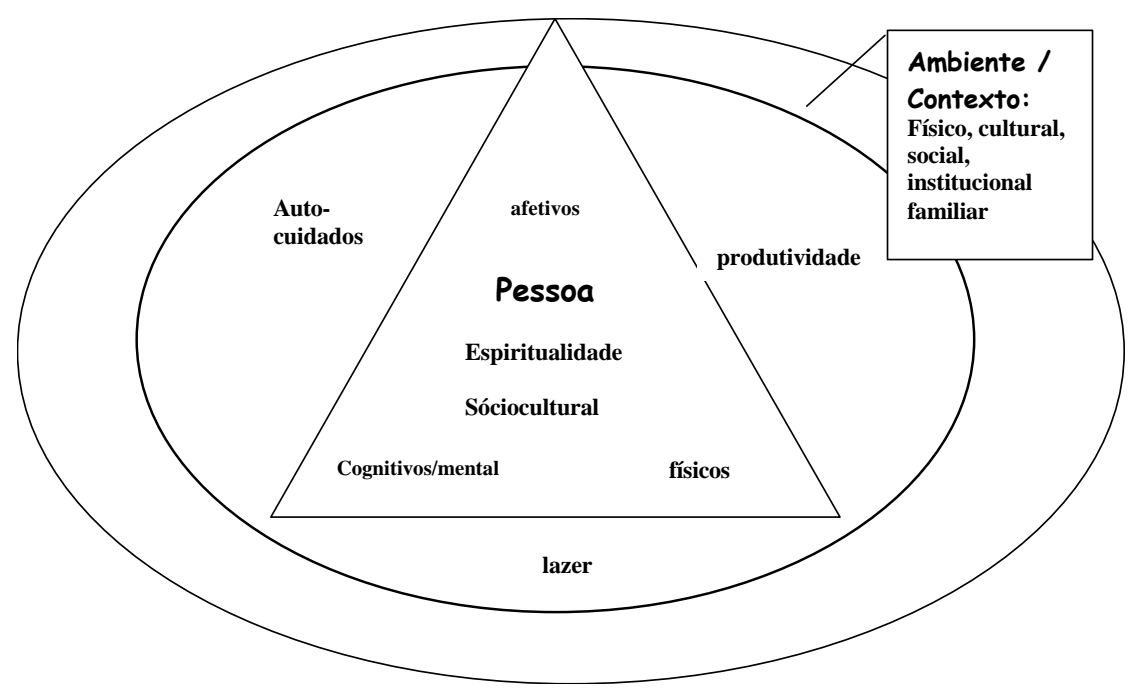

Figura 1 - Aspectos a serem contemplados nos projetos terapêuticos 
Entendemos que essas proposições, tomadas em uma apreensão mais geral, colocam questões fundamentais a serem consideradas no desenvolvimento de projetos terapêuticos: a centralidade da ação na pessoa, o estabelecimento da relação de parceria entre terapeuta e cliente, a validação do protagonismos dos clientes e a construção de projetos a partir de composições sempre variáveis e que respeitem a singularidade de cada situação, indicam inovações conceituais e práticas que rompem com os modelos tradicionais de cuidado e reabilitação e incorporam a crítica aos procedimentos estandartizados e diretivos. Por outro lado, a introdução de um contrato, e da temporalização do processo terapêutico, por meio da definição de objetivos a serem alcançados em certo tempo, associada à avaliações periódicas, conjuntas e participativas, ao nosso ver agregam objetividade e até mesmo uma posição ética adequada à uma real responsabilização, rompendo com a imponderabilidade de processos intermináveis que, no geral, podem colocar a pessoa num circuito negativo de dependência institucional e de indefinição quanto aos objetivos e duração dos processos interventivos. Para os terapeutas, colocar-se em uma relação de parceria e de responsabilização dessa forma nos parece um caminho muito interessante e oferece múltiplas possibilidades de desenvolvimentos de projetos.

\section{As inovações propostas pela desinstitucionalização italiana}

O outro campo de interesse, ativo em nossa perspectiva de trazer inovações para as orientações em terapia ocupacional, está localizado na experiência desenvolvida pelos autores da desinstitucionalização italiana. Como muitos conhecem, essa experiência rompeu com a lógica segregativa e violenta da institucionalização psiquiátrica e passou a propor que a assistência em saúde mental fosse desenvolvida no contexto de uma rede de serviços substitutivos ao manicômio, que passassem a validar os direitos dos usuários, a disponibilidade dos serviços e a priorização de investimentos nos projetos de vida dos usuários, na valorização das pessoas e aumento de seu poder contratual.

Essas mudanças foram associadas, pelos autores, ao imperativo da desconstrução dos aparatos teóricos e institucionais que edificam e legitimam a psiquiatria e se desenvolveram num contexto de críticas ao papel segregador e violento das instituições, o alto custo dos pacientes internados, a eficácia dos tratamentos ambulatoriais e especialmente, a luta pela extensão dos direitos sociais. (ROTELLI et al., 1990; BARROS, 1994; TYKANORI, 1996)

No Brasil, a influência dos pressupostos teóricos e práticos desenvolvidos pela experiência italiana tem crescido, especialmente na última década, trazendo aportes significativos para o processo de reforma psiquiátrica. A discussão sobre o tipo de serviço capaz de promover o cuidado substitutivo, se coloca como central para o planejamento das redes de serviços de saúde mental. Nessa perspectiva, o estabelecimento de novas modalidade de serviço e especialmente os serviços de tipo CAPS III - com funcionamento 24 horas e aos finais de semana e oferta diferenciada de hospitalidade noturna e diurna, e os Serviços Residenciais Terapêuticos - em implementação em todo o país, representam os principais pontos de avanço do processo de superação da institucionalização de tipo asilar e embora ainda insuficientes diante das necessidades da população já demonstram, nas cidades onde são implementados, a possibilidade concreta de superação da internação psiquiátrica. (SARACENO, 1998; BRASIL, 2001)

No contexto da produção dessas transformações as identidades tradicionais dos técnicos e a cisão entre as diversas disciplinas são, permanentemente, colocadas em questão, e a invenção de novas forma de cuidar e responsabilizar-se pelas pessoas com transtorno mental severo se coloca como um desafio central da construção da Política de Saúde Mental, e de todas as categorias profissionais atuantes nesse campo. Dessa forma, a assistência, o ensino e a pesquisa devem assumir o compromisso com o desenvolvimento das inovações teórico, técnicas e políticas exigidas em tal empreitada. (SARACENO, 1999; MÂNGIA; NICÁCIO, 2001)

\section{Nosso diálogo}

Dos referenciais apontados, selecionamos aspectos para a discussão, que nos parecem relevantes e merecem atenção especial quando nos colocamos a tarefa da reflexão interessada na formulação de eixos orientadores para a prática da terapia ocupacional em saúde mental. É claro que se tratam de diretrizes válidas para o conjunto das práticas em saúde mental, mas que podem adquirir contornos específicos e singulares se orientadas para a constituição de práticas inovadoras em terapia ocupacional.

\section{A centralidade da pessoa e do processo de construção/reconstrução de histórias de vida}

Ao assumir como objeto da ação terapêutica a 
pessoa e suas necessidades e não a doença e os sintomas da crise, a ação terapêutica se complexifica e deve buscar apreender e investir não mais na contenção dos sintomas, mas na criação de possibilidades de transformação, crescimento e mudanças capazes de redimensionar a situação existencial da pessoa, em seu conjunto.

Dessa forma, a intervenção deve se constituir como processo complexo que busca propiciar a construção/reconstrução das histórias de vida vulnerabilizadas em processos e percursos singulares, que envolvem a multidimensionalidade de fatores individuais, familiares, sociais, afetivos, emocionais, materiais e tantos outros, que resultam em rupturas importantes que acabam por levar a pessoa a buscar o recurso assistencial como alternativa final (DELL'ACQUA, MEZZINA, 1991).

\section{serviço}

2. O novo tipo de relação cliente-terapeuta-

As mudanças presentes na redefinição do papel e sentido do processo terapêutico implicam no estabelecimento de um novo tipo de relação com aqueles que buscam respostas às suas necessidades, nos serviços de saúde mental. O serviço deve passar a ser considerado como o contexto especial onde ocorre o encontro entre a demanda colocada pelos usuários e a capacidade de resposta e articulação dos recursos dos técnicos e do conjunto do aparato institucional. Tem sido proposto pelos autores da desinstitucionalização italiana, que o serviço deva "assumir a responsabilidade" sobre a saúde mental de um determinado território, tornando-se um elemento ativo na promoção e no cuidado em saúde mental. Ao serviço caberia "ativar recursos" e "promover relações" que forneçam respostas às necessidades e a melhoria da qualidade de vida das pessoas, considerando o conjunto de tensões presentes na polarização entre o cuidado e o controle social e a necessária significação/resignificação contínua do que se considera necessidades (DELL'ACQUA; MEZZINA, 1991; SARACENO,1998, 1999).

Tal "tomada de responsabilidade" implica na invenção de estratégias adequadas à cada situação singular e um tipo de circulação dinâmica que tende a não cristalizar papéis e ações institucionais que se reproduzem, freqüentemente, nas rotinas institucionais estanques e pré-definidas que buscam encaixar a demanda na oferta rotineira do serviço, o que provoca, inevitavelmente, o desencontro, ou muitas vezes a incompatibilidade entre a demanda dos usuários e a oferta dos serviços.

\section{O deslocamento do locus da ação da instituição para o território e para as situações da vida real}

Essa nova forma de responsabilizar-se e produzir projetos terapêuticos singulares deve, vislumbrar um descentramento da ação, num movimento que prioriza ações extra institucionais e potencializa recursos presentes no contexto dos sujeitos em seus territórios existenciais concretos e simbólicos.

Se a crítica aos modelos tradicionais de cuidado e reabilitação recai, fundamentalmente, sobre o excesso de institucionalização e nas rupturas, vulnerabilização e perdas ocasionadas pelos processos de institucionalização permanente.

Se o ideal da inclusão social aparece como a meta mais difícil de ser atingida para aqueles que foram alvo de processos institucionais que totalizam a resposta terapêutica a uma existência vivida entre muros.

Se os novos projetos de cuidado devem se pautar pelo princípio da inclusão social.

Então, podemos aceitar que os projetos terapêuticos inovadores devem evitar a construção de processos que tendam a institucionalizar as necessidades ou a respondê-las a partir de ofertas institucionais muito restritivas, pontuais ou simplificadas. Tais projetos devem assumir um tipo especial de relação com os usuários, colocando-se em interação permanente com suas condições e necessidades objetivas, articulando respostas que possam potencializar e reforçar as relações de interdependência positivas, para que sejam evitados os percursos de institucionalização, e serem favoráveis ao aumento do poder contratual dos sujeitos vulneráveis, a partir dos recursos existentes nos espaços reais das pessoas e em suas redes sócio relacionais.

\section{A nova concepção de projeto terapêutico e a ruptura com a tradicional separação entre prevenção, tratamento e reabilitação}

A ação terapêutica ao situar-se, de forma interativa, na relação entre sujeito e contexto deve lidar com um amplo conjunto de aspectos presentes na vida cotidiana da pessoa: práticos, concretos, simbólicos, relacionais, materiais, etc. De forma a produzir movimentos capazes de oferecerem suportes, proteção e resolução de problemas que contribuam para a superação da situação existencial que tornou possível a manifestação da crise ou a permanência do sujeito em uma situação de muita fragilização e vulnerabilidade em relação ao seu contexto sócio relacional.

Tal dinâmica, representa uma ruptura teórica e prática importante em relação as tradicionais formas de 
cuidado que, para se realizarem, promovem a artificial separação entre sujeito e contexto e limitam a ação terapêutica aos espaços institucionais fechados e prédefinidos dos grupos terapêuticos, grupos de atividade ou dos atendimentos individuais, nos quais, no geral se espera que o indivíduo desenvolva recursos psicoafetivos ou cognitivos para superar sua complexa situação de desvantagem, e os transfira para fora do espaço institucional. Para as pessoas com transtorno mental severo e persistente, tais práticas, no geral, passam a reproduzir a própria situação de empobrecimento e/ou falência existencial que as levou ao serviço, caracterizando aquilo que Saraceno compreende como "prática do entretenimento", na medida em que a expectativa de que a pessoa "transfira habilidades" raramente se concretiza (SARACENO, 1999).

A tradição terapêutica que separa sujeitos e contextos tende também a separar as fases do processo terapêutico, de forma a compreender prevenção, tratamento e reabilitação como fases distintas e consecutivas. Nessa nova perspectiva tal separação deixa de ter sentido e dá lugar a um modelo de intervenção dinâmico e interativo que, ao centrar o olhar no sujeito em seu contexto e na multidimensionalidade de suas necessidades, e não mais na doença e nos sintomas, busca multiplicar os espaços relacionais e as possibilidades de trocas e negociações como estratégia para operar transformações, contínuas e processuais, na trajetória de vida que levou a pessoa ao serviço.

Esse modelo considera também que é na ativação dos espaços relacionais reais que o sujeito restabelece sua contratualidade de cidadão e de produtor de sentido para a sua própria vida e em seu contexto sócio-familiar. Dessa forma, as ações de prevenção, assistência e reabilitação, antes vistas como momentos separados do processo terapêutico, passam a ser integradas e podem acontecer concomitantemente, de acordo com a singularidade de cada processo (SARACENO, 1998, 1999).

\section{A importância da definição de metas significativas, construídas, explicitadas e compartilhadas com os usuários}

É fundamental que tais mudanças práticas e conceituais, ao resignificarem os lugares e papéis tradicionalmente atribuídos aos usuários e técnicos de saúde mental, possam desenvolver projetos e processos compartilhados e em parceria com os sujeitos. A identificação e a reflexão sobre as necessidades deve ocorrer num processo contínuo e conjunto com todos os atores envolvidos no processo e, especialmente, com a pessoa sobre a qual está centrada a ação terapêutica.

A construção dos projetos terapêuticos deve ser compartilhada e os processos desencadeados dotados de sentido e situados na continuidade da vida da pessoa e não representarem rupturas existenciais e práticas, tão comuns nos processos institucionais que separam o "tempo da doença", administrado indefinidamente pelos serviços. (DELL'ACQUA; MEZZINA, 1991). Os processos terapêuticos devem possibilitar a reapropriação contínua, por parte da pessoa, de sua existência cotidiana e não a manutenção da tutela e dependência institucional.

\section{O estabelecimento de critérios avaliativos e o desenvolvimento da avaliação continuada dos processos}

Um último fator, em nosso entendimento, fundamental para a formulação de novas estratégias em saúde mental, reside na construção de parâmetros e critérios que possam sustentar a escolha das práticas e a avaliação dos procedimentos adotados pelos serviços. O campo da avaliação de serviços de saúde mental, ainda pouco difundido em nosso país, requer o desenvolvimento de metodologias qualitativas sensíveis às novas mudanças da assistência em saúde mental, e que possam contribuir com a consolidação de serviços substitutivos. É importante sensibilizar técnicos e gestores para a adoção de estratégias avaliativas, desde o processo de implantação dos novos serviços, que busquem definir indicadores, comuns a um certo conjunto de serviços, que possam nortear as ações institucionais.

De outro lado, é possível pensar que as práticas em curso nos serviços, e especialmente àquelas desenvolvidas por terapeutas ocupacionais, possam ser qualificadas ao introduzirem a perspectiva avaliativa, que contemple a necessária participação dos usuários. A construção de projetos em parceria, requer a definição de metas compartilhadas e aceitas pelos usuários e a definição de percursos a serem desenvolvidos em certo tempo. É freqüente a perda da dimensão temporal nos processos institucionais, que acabam obrigando os usuários a intervenções de longa duração que, muitas vezes, são desprovidas de sentido para a pessoa. Observamos, freqüentemente, nos serviços de saúde mental a reprodução de processos atemporais que tendem a aumentar a dependência institucional ao invés de promover mudanças favoráveis à autonomia da pessoa.

É importante que esse problema, ao nosso ver ético 
É importante que esse problema, ao nosso ver ético e técnico, possa ser superado e acreditamos que a adoção de um contrato de trabalho, entre usuário e serviço, que assuma também a definição compartilhada de metas associadas ao tempo de desenvolvimento das ações propostas, já possa trazer um diferencial importante ao desenvolvimento dos projetos. Projetos que possam ser continuamente avaliados conjuntamente e redefinidos, na medida das necessidades de cada momento, devem constituir a diretriz central dos serviços substitutivos.

MÂNGIA EF. Contributions of the canadian's approach "Client-centered occupational therapy" and of the italian's deinstitutionalization authors for the occupational therapy in mental health. Rev. Ter. Ocup. Univ. São Paulo, v.13, n.3, p.127-34, set./dez. 2002.

\begin{abstract}
The construction of new models for the occupational therapy in mental health is based in dialogue with the propositions's context of the Politics of Mental Health and especially with the introduction of the substitutives services. In this article, starting from the propositions of the "client-centered occupational therapy" and of the Italian's deinstitutionalization authors, we looked for build a dialogue that can guide the adoption of approaches and guidelines for the technical actions of the area. As central points of a new orientation highlighted: 1 . the client-centred practice and of the process of construction/reconstruction of life histories; 2. the new type of relationship client-therapist-service; 3 . the displacement of the locus the action of the institution for the territory and for the situations of the real life; 4 . the new conception of therapeutic project and the rupture with the traditional separation among prevention, treatment and rehabilitation; 5. the importance of the definition of significant goals, built, explicited and shared with the users; 6 . the institution of criteria to evaluate and the development of the continuous evaluation of the process.
\end{abstract}

KEYWORDS: Occupational therapy/trends. Nondirective therapy. Occupational therapy/manpower.

\title{
REFERÊNCIAS
}

BARROS, D. D. Jardins de Abel - desconstrução do manicômio de Trieste. São Paulo: Lemos/EDUSP, 1994.

BRASIL. MINISTÉRIO DA SAÚDE. III Conferência Nacional de Saúde Mental - Cadernos de Textos. Brasília, 2001.

DELL'ACQUA, G.; MEZZINA, R. Resposta à crise: estratégia e intencionalidade da intervenção no serviço psiquiátrico territorial. In: DELGADO, J., org. A loucura na sala de jantar. São Paulo, 1991.

FEARING, V. G.; CLARK, J.; STANTON, S. The clientcentred occupational therapy process. In: LAW, M. (org.) Client-centered occupational therapy. New York: Slack, 1998.

LAW, M., org. Client-centered occupational therapy. New York: Slack, 1998.

LAW, M.; BAPTISTE, S.; MILLS, J. Client-centred practice: what does it mean and does make a difference? Can. J. Ocup. Ther., v. 62, n. 5, p. 250-6, 1995.

MÂNGIA, E.F. Terapia ocupacional: práticas, discursos e a questão da legitimidade científica. Rev. Ter. Ocup. Univ. São
Paulo, v. 10, n. 2/3, p. 60-8, maio/dez., 1999.

MÂNGIA, E.F.; NICÁCIO, M.F. Terapia ocupacional em saúde mental; tendências e principais desafios contemporâneos. In: DE CARLO, M. M. R. P.; BARTALOTTI, C. C. (org.). Terapia ocupacional no Brasil. Fundamentos e perspectivas. São Paulo: Plexus, 2001.

ROTELli, F.; LEONARDIS, O.; MAURI, D. Desinstitucionalização, uma outra via. In: ROTELLI, et al. Desinstitucionalização. São Paulo: HUCITEC, 1990.

SARACENO, B. Libertando identidades. Da reabilitação psicossocial à cidadania possível. Belo Horizonte: Instituto Franco Basaglia/TeCorá, 1999.

SARACENO, B. A concepção de reabilitação psicossocial como referencial para as intervenções terapêuticas. Rev. Ter. Ocup. Univ. São Paulo, v. 19, n. 1, p. 1-44, jan./abr., 1998.

TYKANORI, R. Contratualidade e reabilitação psicossocial. In: PITTA, A. (org.). Reabilitação psicossocial no Brasil. São Paulo: Hucitec, 1996. p. 55-9.

TOWNSED, E.; BRINTNELL, S.; STAISEY, N. Developing guidelines for client-centred occupational therapy practice. Can. J. Occup. Ther., v. 57, n. 2, p. 69-76, 1990. 